\title{
Evaluation of Post Processing Analysis to determine Optimal Thermo-Optical Properties for Adaptive Glazing Systems with Quick Adaptation Speed
}

\author{
Martina Pelle $^{1}$, Francesco Causone ${ }^{1}$, Fabio Favoino ${ }^{2}$, Francesca Contrada ${ }^{3}$, Andrea Kindinis ${ }^{3}$ \\ ${ }^{1}$ Department of Energy, Politecnico di Milano, Via Lambruschini 4, Milano, Italy \\ ${ }^{2}$ Department of Energy, Politecnico di Torino, C.so Duca degli Abruzzi, Turin, Italy \\ ${ }^{3}$ Université Paris-Est, Institut de Recherche en Constructibilité, ESTP, F-94230, Cachan, France
}

\begin{abstract}
Adaptive envelope technologies are considered as ones of the most promising for reducing the energy use in buildings. Nonetheless, their real-world implementation is low, because of fragmented researches and limited capabilities of current BPS (building performance simulation) tools in evaluating properly their behaviour at a time-scale consistent with the highly dynamic drivers effecting the adaptation mechanism. The aim of this research is to investigate the effectiveness of simplified methods to determine optimal thermo-optical properties for adaptive glazing systems able to modulate two parameters, $g$-value (or SGHC) and $\tau_{\text {vis }}$, with quick adaptation speed (5 minutes), using inverse approach and post processing analysis. This type of study is meant to provide a useful tool in early design phase for choosing the best fitted adaptive technology and for the development of new adaptive glazing technologies, since it identifies the requirements to be satisfied by means of the best suited technological solutions.
\end{abstract}

\section{Introduction}

According to IEA-ECBCs Annex 44, adaptive envelope technologies are considered as ones of the most promising technologies for the minimization of energy use in buildings (Heiselberg et al., 2006). As defined by Loonen et al. (2015), an adaptive façade is a "multifunctional system able to change its functions, features or behaviour in response to transient performance requirements and boundary conditions, with the aim of improving the overall building performance”. The design and assessment of adaptive technologies is not a trivial task: they can react, with specific changes in their microscopic or macroscopic configuration, to different drivers (such as temperature, solar radiation or voltage). The adaptivity of a technology needs to be assessed at multiple scales (material, component, building scale) (Attia et al., 2018) and, moreover, the choice of a new typology of building components requires a holistic approach (including aesthetical, structural, economical, and energy efficiency issues). Building Performance Simulation (BPS) represents a useful tool in early-design stage, enabling decision-making about envelope technologies by results comparison. However, current BPS tools have limited capabilities in simulating adaptive behaviour.

An important issue for the evaluation of a new adaptive technology is the assessment of the best suited responsive design characteristics and control strategy: these two aspects may have a strong mutual correlation (Jin et al., 2017) and considering them separately can lead to suboptimal results. Even if some building performance simulation (BPS) tools allow users to model specific adaptive glazing technologies, they are still limited in considering more general adaptive behaviour and they fail in enabling the assessment of both the previous aspects at the same time (Serra et al., 2010).

The assessment of the simulation strategy presents two main problems: 1) the proper time scale for the resolution of the optimisation problem must be established according to the technology adaptation time scale; 2) the thermal history of the building should be preserved from one time-step to the subsequent one. BPSs offer quite scarce possibilities to control properly both these aspects at once and the assessment of adaptive technologies with quick adaptation speed (i.e. on the order of minutes) is possible only for specific technologies. Furthermore, considering the thermal history of the building requires the interaction between several software, and it is normally limited to adaptation time scales greater then one day. To overcome the limited capabilities of the existing tools in modelling and assessing adaptive technologies, various simulation strategies have been adopted in previous researches, with different levels of complexity (Coffey et al., 2010; Asadi et al., 2012). For the analysis of new adaptive technologies, if no existing models are present, workaround and model simplifications are normally used (Loonen et al., 2016). While these studies focus on long adaptation time-scale (days or seasons), in the present paper we investigate a quick adaptation time-scale (5 minutes), following the frequency of the environmental drivers.

The aim of this work is to evaluate the capability of post processing analysis to determine the optimal features of adaptive glazing systems with sub hourly adaptation speed (5 minutes) in two different climates, regarding both visual and thermal behaviour. To do so, we propose a new methodology enabling the optimization of the total energy uses by means of the identification of the most effective range of variability of the glazing thermo-optical properties via post processing technique. In the following paragraphs we provide the description of the developed model, the analysis methodology, and the simulation strategy, highlighting their opportunities and limitations. In this respect, we performed a comparative analysis to evaluate the error committed in evaluating energy uses by 
using a post-processing approach. Finally, as an example of practical application, we assessed the potential energy saving of the optimized systems for all the analysed cases, comparing the annual and seasonal energy uses with the performance of an optimal static technology.

\section{Model description}

The overarching aim of the study is to evaluate the optimal behaviour of an ideal adaptive glazing system able to modulate its thermo-optical properties in response to changing in external or internal drivers (environmental conditions and/or internal loads, i.e. lights, occupancy, equipment), thereby minimizing the total energy use in an indoor space. A typical cellular office room has been simulated in EnergyPlus 9.0, running annual simulations. The physical model consists in an office room with dimensions equal to ( $\mathrm{LxWxH}) 5.3 \mathrm{~m} \times 4.5 \mathrm{~m} \times 4.3 \mathrm{~m}$ and lightweight envelope technologies $\left(\mathrm{U}_{\text {wall }}=0.15 \mathrm{Wm}^{-2} \mathrm{~K}^{-1}\right)$, with one wall facing the outdoor environment and the other walls being adjacent to spaces with similar set point conditions (modelled as adiabatic). The occupancy is of two people fulfilling a typing activity, with metabolic rate of $117 \mathrm{~W} /$ person (ASHRAE, 2009). The equipment power density has been set to $12 \mathrm{~W} / \mathrm{m}^{2}$. The schedule for the occupancy and the electric equipment considers a normal office work day, starting from 9 a.m. to 1 p.m., and from 2 p.m. to 6 p.m. without considering differences between weekends and week days. The lights turn on following the occupancy schedule, from 9 am to 1 p.m. and from 2 p.m. to 6 p.m., every day of the week. The lighting system follows a control strategy based on daylight availability. A minimum illuminance level of 500 lux for typical office activities has been set according to EN 12464-1:2011 during the occupancy schedule. The work plan has been set in the centre of the office room at a height of $0.8 \mathrm{~m}$. Lights switch on, based on a Continuous Dimming Control that ranges from a minimum lighting power density of $0 \mathrm{~W} / \mathrm{m}^{2}$ up to a maximum of $10 \mathrm{~W} / \mathrm{m}^{2}$, when the daylight illuminance level on the work plane is lower than 500 lux, in order to guarantee the minimum illuminance level set by the standard. The heating and cooling systems consist of an Ideal Purchased Air module for the calculation of the energy needs for space heating or cooling. The system has an ideal unlimited operational power. It calculates the ideal loads based on a dual set point, throughout the whole simulation period, $\mathrm{T}_{\min }=20$ ${ }^{\circ} \mathrm{C}$ and $\mathrm{T}_{\max }=26^{\circ} \mathrm{C}$. With the assumption that the heating and cooling plants are powered by an inverter heat pump, with a SCOP $=2$ (Seasonal Coefficient of Performance) for the heating system and a SEER = 3 (Seasonal Energy Efficiency Ratio) for the cooling system, it is possible to evaluate through a post processing analysis, the values of the final energy use. The schedule for the heating and cooling systems is always on from 7 a.m. to 8 p.m., every day of the week.

The study has been conducted analysing the performance of a system operating in different locations: Milan and Paris. According to the Köppen-Geiger Classification, these cities belong to the same warm temperate climate, have the same fully humid precipitation category, but differ about temperatures: Milan has a hot summer (Cfa classification) and Paris has a warm one (Cfb classification) (Köppen and Geiger, 1954). The accumulated temperature differences of the two locations have been analysed, according to ISO 15927-6:2007. The analysis highlights that Milan has a warmer heating period and hotter summer than Paris (see Table 1).

\section{Transparent components}

Regardless the adaptive mechanism, the behaviour of ideal adaptive glazing technologies can be described through their capability to modulate two parameters: $g$ value (or SGHC) and $\tau_{\text {vis }}$ (Favoino et al., 2015). Thus, to evaluate the adaptive behaviour of the glazing system through post processing analysis, a series of models with static windows, with dimension ( $\mathrm{LxH}) 2.6 \mathrm{~m} \mathrm{x} 2.7 \mathrm{~m}$, have been created. The WWR (window to wall ratio) of the external façade is $60 \%$. The U-value of the glazing systems has been set at $1.8 \mathrm{~W} / \mathrm{m}^{2} \mathrm{~K}$ for both cities, according to the prescription of the Italian legislation for Milan (DM 162/2015, Appendice A), which is the stricter between the two national standards. Each static window corresponds to a specific state of the adaptive glazing system. The analysed modulating adaptive range for $g$ value and $\tau_{\text {vis }}$ have a minimum value of 0.1 and a maximum of 0.9 , with steps of 0.1 (Table 2). Among all the possible combinations of the two parameters, only those physically reasonable have been retained. For this purpose, the luminous efficacy $K_{e}$ has been considered. It is calculated as the ratio between $\tau_{\text {vis }}$ and $g$-value and gives the amount of visible radiation compared to the total amount of solar energy transmitted through a glazing. Referring the luminous efficacy to the spectral selectivity of the glazing, the theoretical maximum for $K_{e}$ is equal to 2.41: this values indicates that only visible radiation is transmitted into the building and it is limited by the ratio of the energy contained in the solar visible spectrum compared to the whole solar spectrum at the sea level, which is approximately $41.5 \%$ (Favoino et al., 2015). Thus, among 81 possible combinations of the two parameters, only 67 solutions have been retained for the analysis, which are the ones satisfying the requirement:

$$
K_{e}=\frac{\tau_{\text {vis }}}{g \text {-value }} \leq 2.41 \quad[-]
$$

It is worth noticing that assuming a varying $g$-value and $\tau_{v i s}$ and a fixed U-value is a simplification of the real physical behaviour of the glazing system. However, this simplification was required to reduce the complexity of

Table 1: Degree days for heating and cooling period in Milan and Paris.

\begin{tabular}{|l|c|c|c|}
\hline & Heating period & HDD & CDD \\
\hline Paris Orly (IWEC) & $15 / 10-15 / 04$ & 2557 & 8 \\
\hline TMY Milano 05-16 & $15 / 10-15 / 04$ & 2099 & 156 \\
\hline
\end{tabular}

Table 2: Glazing system thermal transmittance and thermo-optical modulating ranges.

\begin{tabular}{|c|c|c|c|c|c|}
\hline & $\begin{array}{c}\mathbf{U} \\
{\left[\mathbf{W} \mathbf{m}^{-2} \mathbf{K}^{-1}\right]}\end{array}$ & \multicolumn{2}{|c|}{$\tau_{\text {vis }}[-]$} & \multicolumn{2}{|c|}{ g-value [-] } \\
\hline \multirow{2}{*}{$\begin{array}{l}\text { Glazing } \\
\text { system }\end{array}$} & \multirow{2}{*}{1.80} & {$[\min -\max ]$} & [step] & {$[\min -\max ]$} & [step] \\
\hline & & [0.1-0.9] & {$[0.1]$} & [0.1-0.9] & {$[0.1]$} \\
\hline
\end{tabular}


the model in terms of number of simulated cases and, consequently, the time required for the simulations. Further analysis might allow, in future works, to consider also the effects of a varying U-value.

\section{Methods}

\section{Analysis approach}

To address the assessment of adaptive components two main analysis approaches can be implemented: the direct or traditional one and the inverse one (Zeng et al., 2011). In the direct approach, the properties and characteristics of the adaptive technology are defined first, then a model is created, and the performance is evaluated and optimized. Generally, the adaptive system performance is compared to a state-of-the-art reference technology to assess the energy saving potential. The inverse approach enables, instead, the investigation of optimal adaptive properties as a general problem, which requires that the system can freely assumes the adaptive state that best fits the achievement of the optimization target. This approach aims to define the optimal properties of the building envelope by minimizing or maximizing an objective function which could be either the energy consumption or the indoor environmental comfort. For the purpose of this study, the inverse approach results to be the best suited.

\section{Simulation strategy}

To overcome the limited capabilities of the existing tools in the assessment of general-purpose problems concerning adaptive technologies, a simplified simulation strategy has been used, using an off-line post-processing technique. The adopted simulation strategy consists in simulating different models separately for the whole simulation period (one year), each of them having a glazing system with steady thermo-optical properties, which represent one possible states of adaptation (Figure $1,(1)$ ). The simulation time step has been set at 5 minutes. From an annual simulation, three months have been selected and analysed separately: January, April and July, each representative of different seasons. For each case, the four main exposure (N, S, E, W) have been considered separately in the two chosen locations: Milan and Paris. An off-line, post processing analysis has been performed on the collected data using the software MATLAB ${ }^{\mathrm{TM}}$, with the aim of minimizing an objective function given by the sum of the energy uses for space heating, cooling and lighting at each time step $t$, as follows:

$$
\min \left\{\begin{array}{c}
f(X)=E_{p}=E_{\text {use,cool }}+E_{\text {use, heat }}+E_{\text {use,light }}[k W h] \\
X(t)=\left(g-\operatorname{value}(t), \tau_{\text {vis }}(t)\right)
\end{array}\right.
$$

The evaluation of adaptive performance throughout postprocessing method enables to detect instantaneous switch of façade states, making it suitable for the assessment of technologies with faster adaptation speed. Furthermore, it allows a more accurate control of the optimization function, that can be chosen and formulated directly by the user. For each time step (i.e. every 5 minutes), the minimum value of the total energy use has been found among the different steady glazing systems. The thermooptical properties related to the minimum energy use have been detected and the evolution of the internal building variables have been rebuilt through the combination of the optimal situation at each time step, as in a patchwork (Figure 1, (2)). The main drawback of this patching adaptive model (PAM) is that it fails in accounting for transient thermal energy storage. Indeed, it does not enable the update of the model physical states at each time step and the initial conditions of a simulation frame are different from the final conditions of the previous one. This may lead to errors in the evaluation of the energy uses of ideal adaptive systems, limiting the reliability of results. To address this issue, we performed a comparative analysis, using the output of the PAM derived from postprocessing analyses. The resulted optimal time-related trend of the two analysed parameters and the electric power for lighting have been used to run simulations that reproduce the real evolution of the physical model with the optimized adaptive glazing system using the EMS (Energy Management System) of EnergyPlus (Figure 1, (3)). The results of this continuously adaptive model (CAM), accounting for transient thermal energy storage, have been compared to the result of the PAM in terms of energy uses, to evaluate the error related to the simplified simulation strategy (Figure 1, (4)).

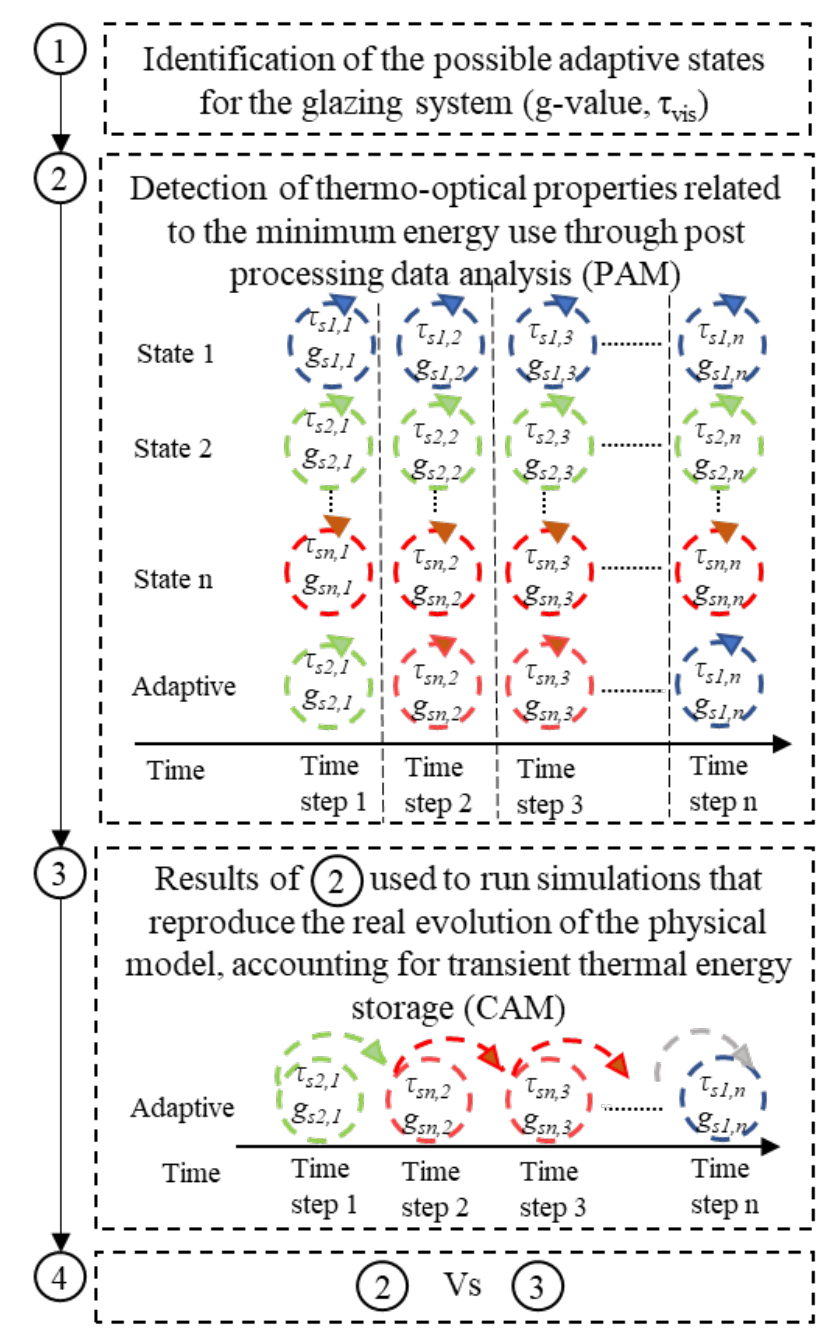

Figure 1: Simulation strategy. 


\section{Results}

\section{Comparative analysis: error estimation}

To assess the error related to the inaccuracies of the simplified post-processing approach, we analysed and compared, for each climate and for the four main exposures, the results of PAM and CAM in terms of energy uses (Figure 1, 4). Figure 2 shows the energy uses derived from the CAM and from the PAM and the percentage error related to the latter for total energy uses, energy uses for heating and for cooling. The energy uses for lighting are not shown since the output of the lighting power, as a function of the daylighting control from the post processing analysis, has been used as input for the CAM. The results show that using the PAM leads to significant error in the energy uses evaluation. The total annual energy uses of the CAM result to be higher than the energy uses derived from the post-processing optimization (i.e. PAM) in all the analysed cases. The higher errors are highlighted in the mid-season periods and the most sun-drenched exposures, with error reaching the maximum of $77 \%$ for the energy uses for cooling in the case of Southern façade in Paris; whereas, during colder period the error committed is lower, of about $10 \%$ for January in each climate and exposure. This means that PAM could be suited for the assessment of optimized adaptive behaviour for heating predominant applications, while it is inappropriate when it comes to the assessment of adaptive façades to be used in warm climates, where, maybe, ruled based control can provide better results.

\section{Energy saving potential of the adaptive glazing}

To assess the effectiveness of the optimized adaptive systems derived from the early analysis, we analysed their energy saving potential. Therefore, for each case, the energy uses resulting from the CAM have been compared with a benchmark. This was chosen as the static glazing technology optimizing the total energy use (eq. 2) for the whole year for each climate and orientation (reported in Table 3). The analysis of the energy saving potential of the optimal adaptive system highlights again the limitations of simplified simulation structures in meeting the reduction of energy uses for cooling. Referring to Figure 3 it is possible to notice that in the cases where the energy uses for cooling are prevalent over the whole year, the optimal adaptive states analysed do not bring any energy use benefit compared to a static glazing, as for the Southern and Eastern exposure in Milan. On the contrary, in Paris, where the energy uses for heating prevails, the energy saving is relevant and range from $10 \%$ to $21 \%$. As a matter of fact, looking deeper at the colder season (January) it is evident that in Milan, with a glazing controlled in the optimized way, we achieve total energy saving between $13 \%$ and $42 \%$ (excluding North exposition). In Paris the energy uses reduction ranges from $7 \%$ to $35 \%$, slightly lower than in Milan due to the limited capability of reducing heating demand by means of solar radiation at these latitudes. These results are remarkable if we consider the WWR of the exposed wall, which is of $60 \%$. Instead, when we look at the warmer
Table 3: Thermo-optical properties of the optimal steady (non-adaptive) technologies by exposures and climates.

\begin{tabular}{|c|c|c|c|c|}
\hline & & $\mathbf{U}\left[\mathbf{W} / \mathbf{m}^{2} \mathbf{K}\right]$ & g-value $[-]$ & $\boldsymbol{\tau}_{\text {vis }}[-]$ \\
\hline \multirow{2}{*}{ East } & Milan & 1.8 & 0.1 & 0.2 \\
\cline { 2 - 5 } & Paris & 1.8 & 0.3 & 0.7 \\
\hline \multirow{2}{*}{ Wouth } & Milan & 1.8 & 0.1 & 0.2 \\
\cline { 2 - 5 } & Paris & 1.8 & 0.2 & 0.4 \\
\hline \multirow{2}{*}{ North } & Milan & 1.8 & 0.2 & 0.4 \\
\cline { 2 - 5 } & Paris & 1.8 & 0.3 & 0.7 \\
\cline { 2 - 5 } & Milan & 1.8 & 0.3 & 0.7 \\
\cline { 2 - 5 } & Paris & 1.8 & 0.3 & 0.7 \\
\hline
\end{tabular}

periods, no energy saving is shown from the adaptive system for the most sun-drenched exposures, south and east, for July and April, which proves the noneffectiveness of the post- processing optimization strategy in determining optimal thermo-optical properties of adaptive glazing for reducing cooling energy use.

\section{Discussion}

The aim of the simulations was to optimize the total energy use enabling the variation of the parameters $g$ value and $\tau_{\text {vis }}$ with time steps of 5 minutes. Beside the energy uses evaluation, the results of the post processing analysis have been analysed assessing the frequency of the occurrence of each adaptive state for $g$-value and $\tau_{\text {vis }}$ and the frequency of the luminous efficacy $K_{e}$, shown in Figure 4. This type of analysis could be very useful for the identification of the most effective range of variability of the thermo-optical properties for a general-purpose problem. Therefore, it can be considered as a tool for the early design phase of new adaptive glazing technologies, since it identifies the most challenging requirements to be satisfied by means of the best suited technological solutions, although it does not provide useful information on the best suited control logic. The results highlight that in in each case more than the $60 \%$ of the actuated adaptive states have a luminous efficacy $\mathrm{K}_{\mathrm{e}}$ higher than 1.00 (Figure 4, on the left), which means that in the analysed climates the adaptive component should be able to maintain the proportion between the infrared and visible part of the spectrum in the transmitted energy or to admit equal amount of infra-red and visible components of the spectrum, minimizing the overheating and favouring the daylighting, when possible. During warm periods (July for both climates and April for Milan) the most frequent adaptive states are related to lower g-values (mostly 0.1), while during cold ones the frequency is higher for $g$-value higher than 0.6. These results are coherent with previous studies conducted using more sophisticated simulation strategies, as in Favoino et al. (2015). Therefore, it is possible to say that an adaptive component, to be effective, needs to assume extreme values of its modulating range (sometimes the extreme values are the most frequent in different seasons for the same exposure) and it needs to be able to modulate its selectivity, i.e. to varying the $g$-value and $\tau_{\text {vis }}$ independently one from another, which is not always possible for the existing adaptive technologies. 

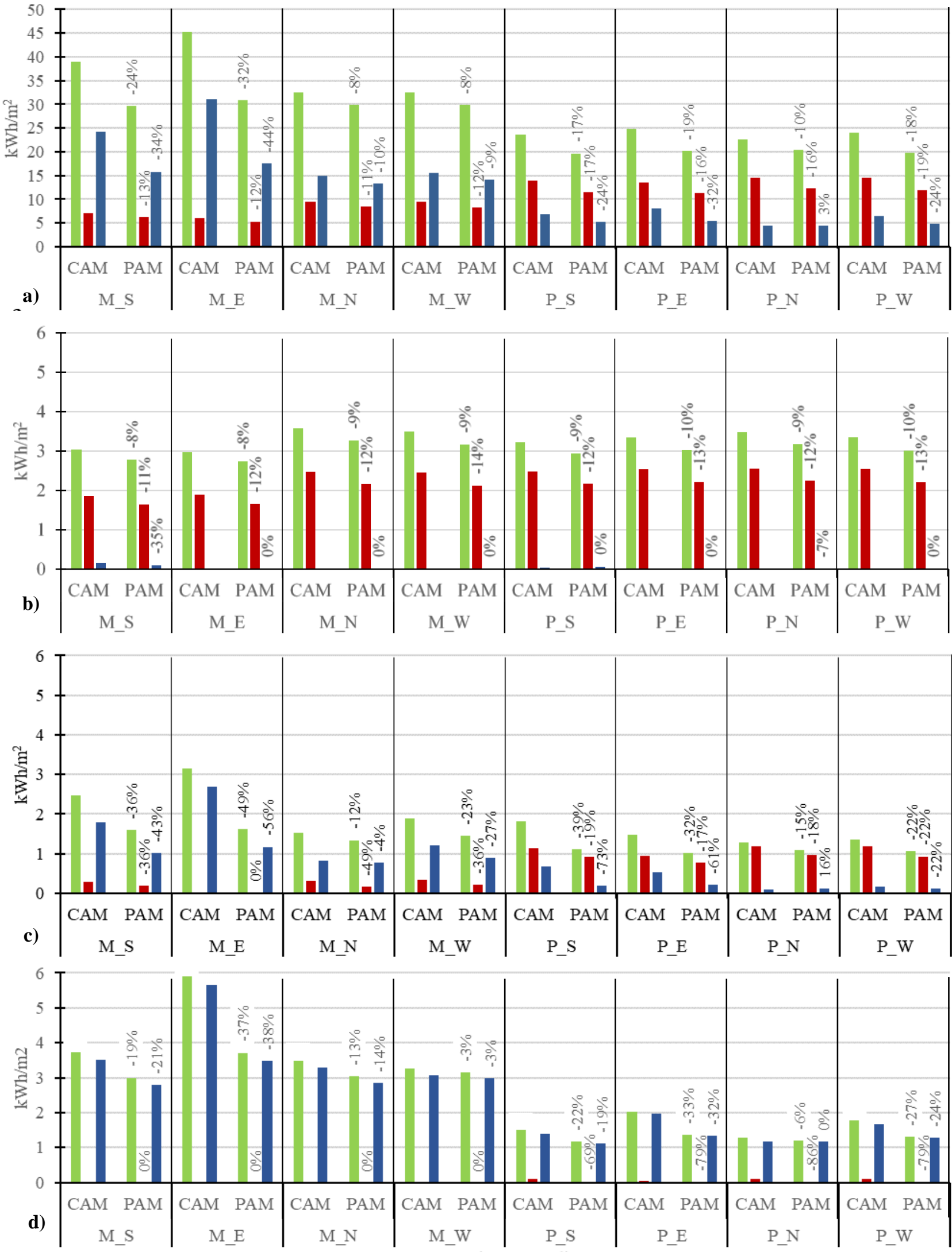

- Tot - Heating Cooling

Figure 2: Comparison of energy uses, broken by purpose (total energy uses (sum of energy uses for heating, cooling and lighting), energy uses for heating and energy uses for cooling), between Patching adaptive model (PAM) and Continuously adaptive model (CAM) for the two analysed climates Milan $(M)$ and Paris $(P)$ and the main four exposure (south (S), east (E), north (N) and west (W)). From the top: a) annual energy uses; b) January energy uses; $c$ ) April energy uses; July energy uses. The percentage represent the error related to the simplified post-processing analysis. 

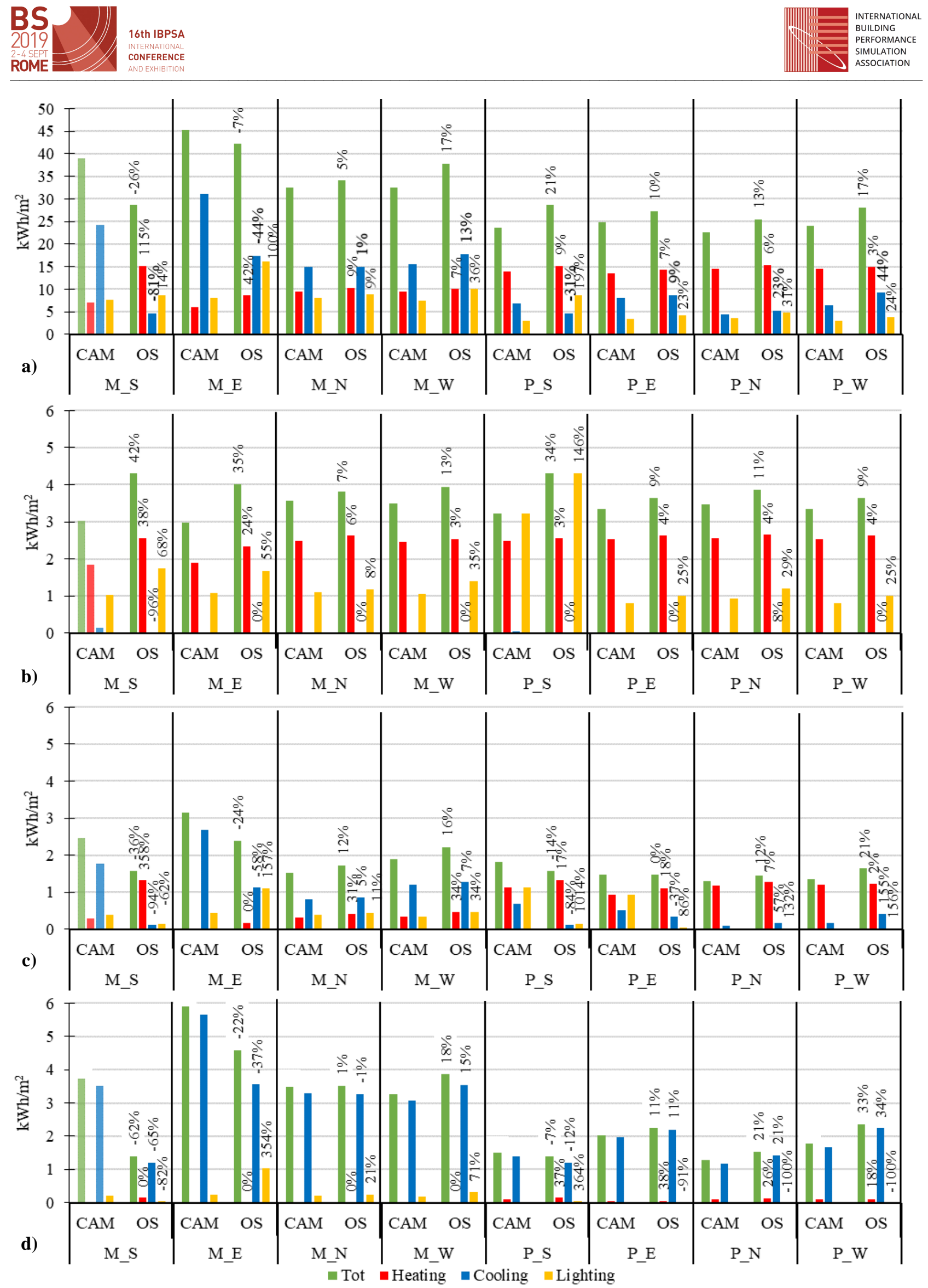

Figure 3: Energy saving, broken by purpose (total energy uses (sum of energy uses for heating, cooling and lighting), energy uses for heating, energy uses for cooling and energy uses for lighting), between Continuously adaptive model (CAM) and optimal steady window (OS) for the two analysed climates, Milan (M) and Paris (P) and the main four exposure (south (S), east (E), north ( $N$ ) and west (W)). From the top: a) annual energy uses; $b$ ) January energy uses; c)

April energy uses; July energy uses. The percentage represent the error related to the simplified post-processing analysis. 

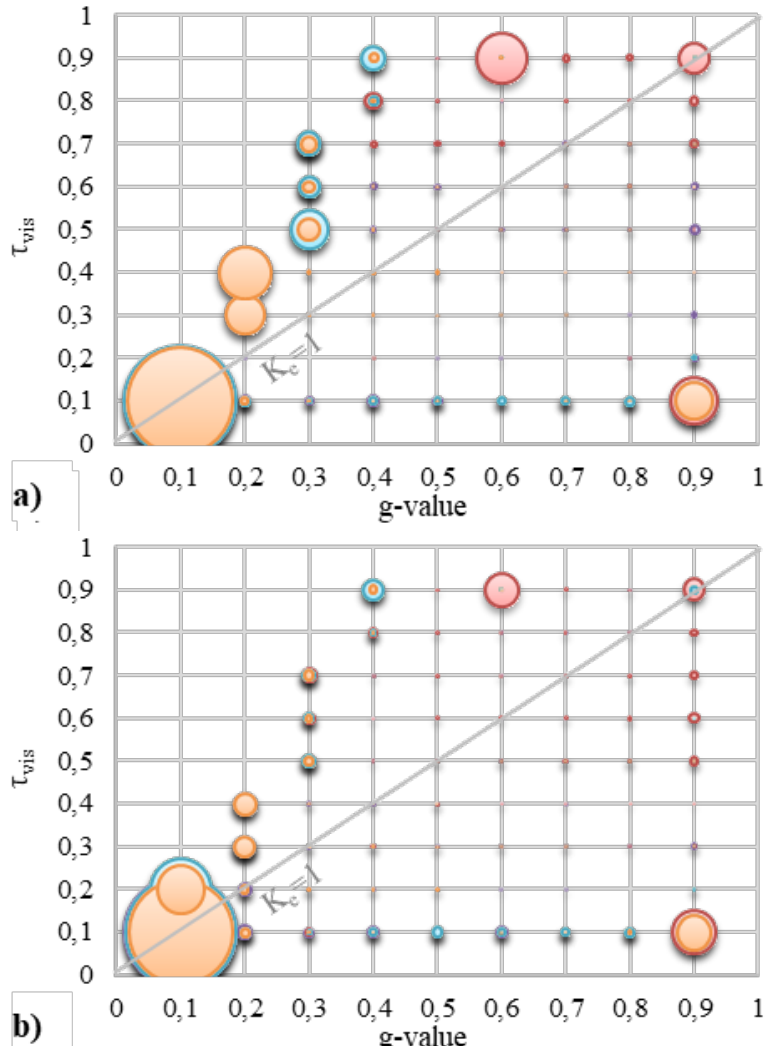

b)
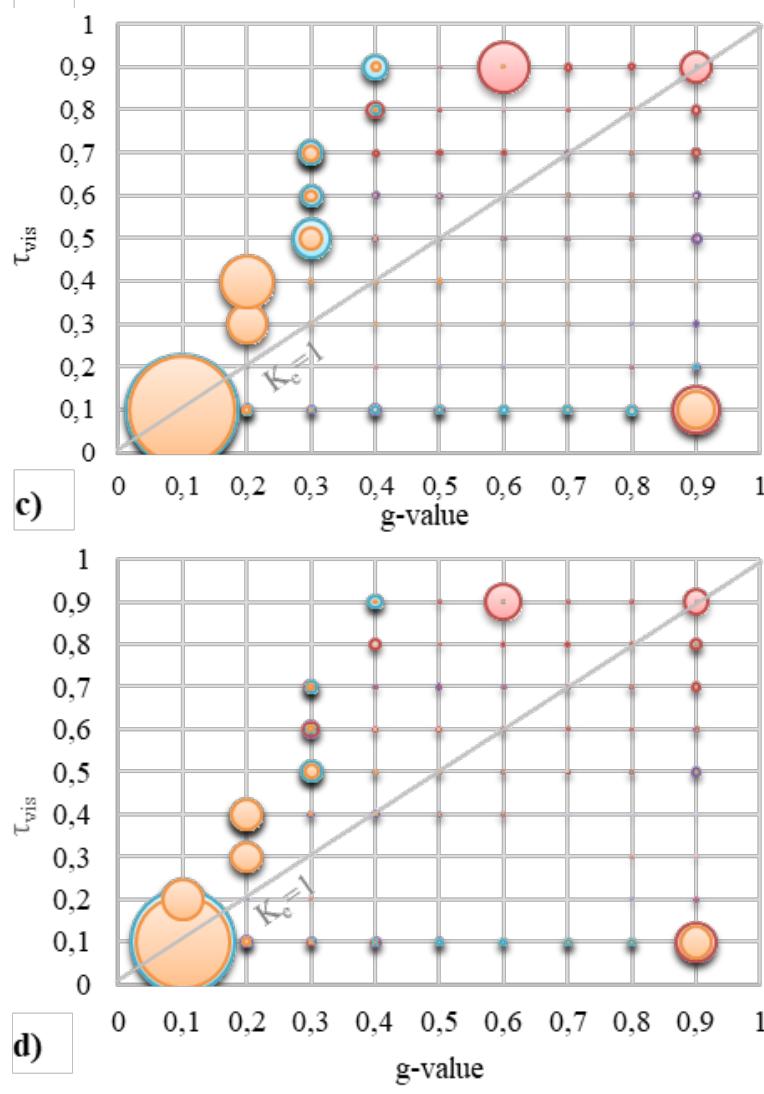

○ Mil Jan O Par Jan ○ Mil Apr ○ Par Apr ○ Mil Jul ○ Par Jul

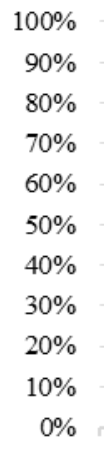
$\begin{array}{llllllllllll}0,10 & 0,35 & 0,60 & 0,85 & 1,10 & \mathrm{~K}_{\mathrm{e}} & 1,35 & 1,60 & 1,85 & 2,10 & 2,35 & 2,41\end{array}$

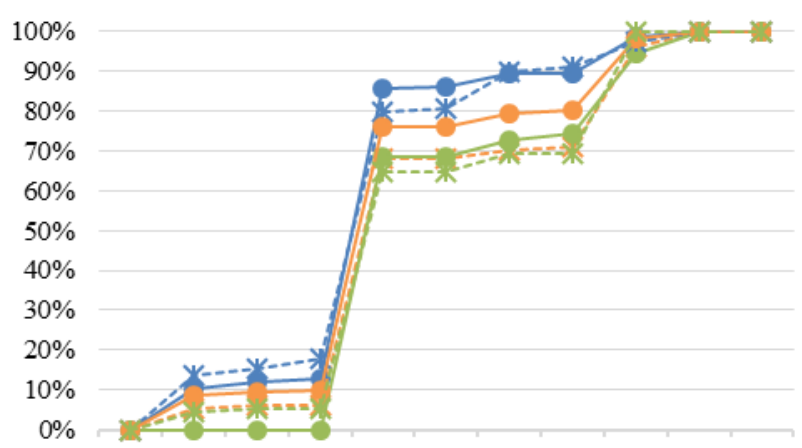

$\begin{array}{llllllllllll}0,10 & 0,35 & 0,60 & 0,85 & 1,10 & 1,35 & 1,60 & 1,85 & 2,10 & 2,35 & 2,41\end{array}$ $\mathrm{K}_{\mathrm{e}}$
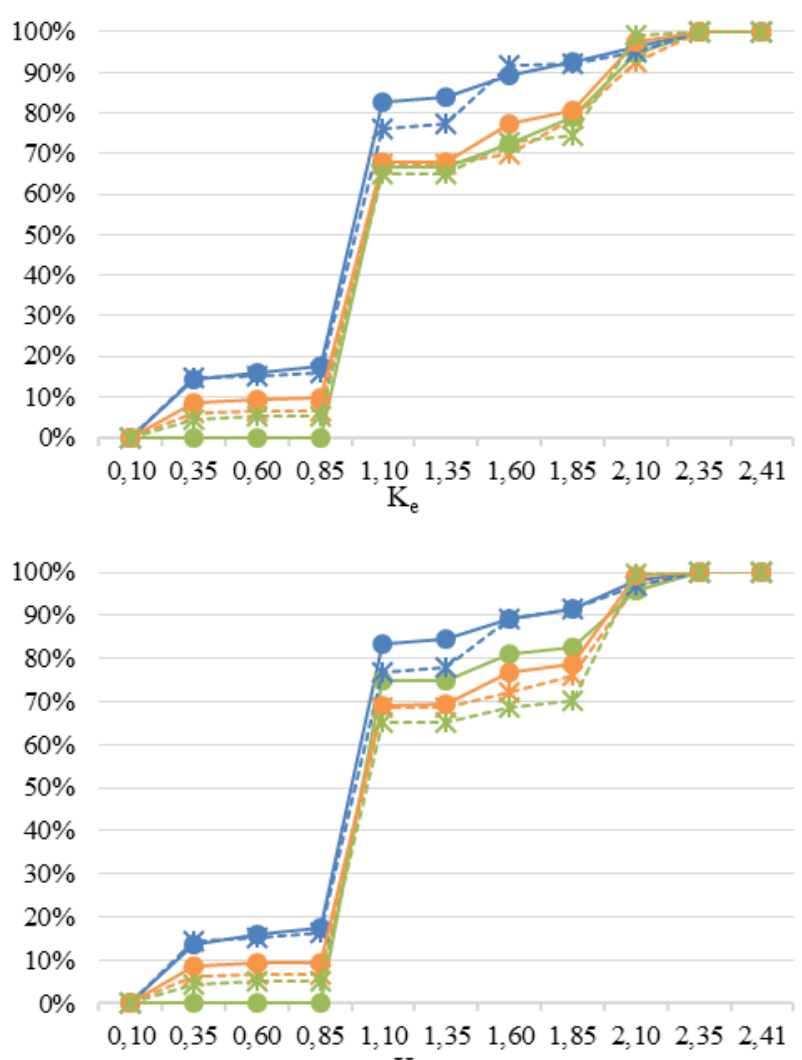

$\begin{array}{lllllllllll}0,10 & 0,35 & 0,60 & 0,85 & 1,10 & 1,35 & 1,60 & 1,85 & 2,10 & 2,35 & 2,41\end{array}$

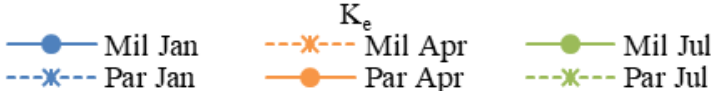

Figure 4: Frequency analysis of the $g$-value and $\tau_{\text {vis }}$ (on the left) and cumulative frequency analysis of the luminous efficacy $K_{e}$ (on the right), broken by climate and month. From the top: a) exposure east; b) exposure south; c) exposure north; d) exposure west. 


\section{Conclusions}

This study was meant to evaluate a simplified method, which uses post processing technique and inverse approach, to determine the optimal features for adaptive glazing system with quick adaptation speed. As a matter of fact, the available simulation tools offer scarce possibilities in evaluating adaptation technologies in early design phase, where several design options need to be compared quickly to address effectively the subsequent design choices. In this study, each yearly optimization takes approximately 15 minutes, running on a $1.8 \mathrm{GHz}$ clock processor, with 16 GB RAM. In order to obtain more reliable results and useful information on the suitable control logic of the adaptive glazing, a method which optimizes glazing properties at each time step during the simulation run-time should be implemented, as done by Favoino et al. (2016), which adopts Receding Horizon Control of the glazing in order to minimize yearly energy uses. Even though, in order to optimize subhourly glazing control, the required numerical effort might be prohibitive (approximately 24h simulation time to optimize sub hourly properties of one day).

The results show that for reducing energy uses for heating and lighting, the post-processing approach seems to be effective, although the results obtained might be suboptimal. On the other hand, the post-processing approach seem to fail in accounting the potential energy use reduction for cooling, hence the optimal glazing properties to obtain such a reduction. Furthermore, it is possible to say that an adaptive component, to be effective, needs to assume extreme values of its modulating range (sometimes the extreme values are the most frequent in different seasons for the same exposure) and it needs to be able to modulate its selectivity, i.e. to varying the $g$-value and $\tau_{v i s}$ independently one from another, which is not always the case of the existing adaptive technologies.

When cooling energy uses are present, simple rule-based control to evaluate the optimal properties of the glazing may be performing as good as adopting Receding Horizon Control optimization, as the objective during the cooling season would be to prevent unwanted solar radiation, beyond what it is strictly needed for daylight. Therefore, future work could be directed in elaborating a mixed method which combines optimization of glazing properties in post processing during the heating season, and optimization based on rule-based control during cooling period. This might yield larger energy use reduction, while better directing performance based adaptive glazing design.

\section{References}

Asadi, E. et al. (2012). A multi-objective optimization model for building retrofit strategies using TRNSYS simulations, GenOpt and MATLAB. Building and Environment 56, 370-378.

American Society of Heating, Refrigerating and AirConditioning Engineers (2009). 2009 ASHRAE Handbook of Fundamentals. American Society of
Heating, Refrigeration and Air-Conditioning Engineers. Atlanta (GA)

Attia, S. et al. (2018). Current trends and future challenges in the performance assessment of adaptive façade systems. Energy and Buildings 179, 165-182.

Coffey, B. et al. (2010). A software framework for model predictive control with GenOpt. Energy \& Buildings 42(7), 1084-1092.

European Committee for Standardization (2011)., Light and Lighting - Part 1: Indoor work places (EN 12464-1)

Favoino, F. et al. (2016). Optimal control and performance of photovoltachromic switchable glazing for building integration in temperate climates. Applied Energy 178, 943-961.

Favoino, F., Overend, M. and Jin, Q. (2015). The optimal thermo-optical properties and energy saving potential of adaptive glazing technologies. Applied Energy 156, $1-15$.

Heiselberg, P. et al. (2006). Integrating Environmentally Responsive Elements in Buildings. Proceedings from the 27th AIVC Conference. Lyon (France), 20-22 November 2006.

Jin, Q., Favoino, F. and Overend, M. (2017). Design and control optimisation of adaptive insulation systems for office buildings. Part 1: Adaptive technologies and simulation framework. Energy 127, 301-309.

Köppen, W., Geiger, R., (1954). Klima der Erde. KlettPerthes. Gotha (Germany).

Loonen, R.C.G.M. et al. (2015). Design for façade adaptability - Towards a unified and systematic characterization. Proceedings from the 10th Conference on Advanced Building Skins. Bern (Switzerland), 03-04 November 2015.

Loonen, R.C.G.M. et al. (2016). Review of current status, requirements and opportunities for building performance simulation of adaptive facades. Journal of Building Performance Simulation 10 (2), 205-223.

Ministero dello Sviluppo Economico (2015). Applicazione delle metodologie di calcolo delle prestazioni energetiche e definizione delle prescrizioni e dei requisiti minimi degli edifici, Appendice A (DM 162/2015)

Serra, V., Zanghirella, F. and Perino, M. (2010). Experimental evaluation of a climate façade: Energy efficiency and thermal comfort performance. Energy and Buildings 42(1), 50-62.

Zeng, R. et al. (2011). New concepts and approach for developing energy efficient buildings: Ideal specific heat for building internal thermal mass. Energy and Buildings 43(5), 1081-1090. 